\title{
Research Article \\ Effective Thermal Conductivity of Open Cell Polyurethane Foam Based on the Fractal Theory
}

\author{
Kan Ankang and Han Houde \\ Merchant Marine College, Shanghai Maritime University, Shanghai 201306, China \\ Correspondence should be addressed to Kan Ankang; ankang0537@126.com
}

Received 6 March 2013; Revised 13 September 2013; Accepted 19 November 2013

Academic Editor: Martha Guerrero

Copyright ( $\odot 2013$ K. Ankang and H. Houde. This is an open access article distributed under the Creative Commons Attribution License, which permits unrestricted use, distribution, and reproduction in any medium, provided the original work is properly cited.

Based on the fractal theory, the geometric structure inside an open cell polyurethane foam, which is widely used as adiabatic material, is illustrated. A simplified cell fractal model is created. In the model, the method of calculating the equivalent thermal conductivity of the porous foam is described and the fractal dimension is calculated. The mathematical formulas for the fractal equivalent thermal conductivity combined with gas and solid phase, for heat radiation equivalent thermal conductivity and for the total thermal conductivity, are deduced. However, the total effective heat flux is the summation of the heat conduction by the solid phase and the gas in pores, the radiation, and the convection between gas and solid phase. Fractal mathematical equation of effective thermal conductivity is derived with fractal dimension and vacancy porosity in the cell body. The calculated results have good agreement with the experimental data, and the difference is less than $5 \%$. The main influencing factors are summarized. The research work is useful for the enhancement of adiabatic performance of foam materials and development of new materials.

\section{Introduction}

Because of distinguished adiabatic performance, open cell polyurethane foam, with small density and low thermal conductivity $(0.018 \sim 0.032200 \mathrm{~W} /(\mathrm{m} \cdot \mathrm{K}))$, is applied in various fields such as building, food cold storage, and refrigerating cargo transportation, for heat conservation purpose. The irregular geometrical construction of open cell polyurethane foam makes it irregular in physical properties. And it makes the theoretic research difficult, especially in the accurate thermal performance. Actually, adiabatic materials' thermal conductivity can be measured by the plate thermal guarded device, but it is inconvenient for science research and the polyurethane foam development. It has been a considerable research project for thermophysics engineering and hylology to analyze and estimate the effective thermal conductivity of porous medium for a long time [1]. While the foam porous medium material is taken as the research project to calculate the thermal conductivity, it is always supposed as the connecting virtual medium in large-scale space, that is, "the average volume" in geometric distribution. Whitaker [2, 3] and Whitaker and Chou [4] took the virtual "average volume" method to describe the procedure of the heat and mass transfer inside the porous medium. The view was taken that the porous medium was combined with solid phase material, liquid, and gas. The gas phase contains dry air and vapor. Supposed that all the phases in porous medium were balances of heat and the pores' dimensions were according to "average volume", a dozen of variables involved in the mathematical formula. Yu et al. [5, 6] also investigated experimentally their coupling and diffusing physical model and derived the relative mathematical formula.

There are two main methods to estimate the thermal conductivity of porous medium materials nowadays. One is that the thermal conductivity is illuminated as the complicated mathematical functions by the pore proportion and the microstructure parameters. Lagarde [7] derived the equivalent effective thermal conductivity $\lambda_{e}$ function of the saturation porous materials. The equivalent effective thermal conductivity is obtain from

$$
\lambda_{e}=\varphi \lambda_{f}+(1-\varphi) \lambda_{s}
$$

where $\lambda_{f}$ is the fluid phase thermal conductivity $\left(\mathrm{Wm}^{-1} \mathrm{~K}^{-1}\right)$ and $\lambda_{s}$ is that of the solid phase $\left(\mathrm{Wm}^{-1} \mathrm{~K}^{-1}\right)$. 
Here the supposition was made that heat fluxes through fluid in the pore and by the solid phase of the porous body were individual and took place simultaneously. However, the heat transfer was also proceeding between the fluid phase and the solid phase simultaneously. So the real model was more complicated than the expression in (1). So Williams and Dawe [8] developed the function as follows:

$$
\lambda_{e}=\varepsilon\left[\varepsilon_{1} \lambda_{f}+\left(1-\varepsilon_{1}\right) \lambda_{s}\right]+\frac{(1-\varepsilon)}{\varepsilon_{2} / \lambda_{f}+\left(1-\varepsilon_{2}\right) / \lambda_{s}},
$$

where $\varphi=\varepsilon \varepsilon_{1}+(1-\varepsilon) \varepsilon_{2}$. The factor $\varepsilon$ is ratio that the heat flux transfers along with the temperature grads to the total heat flux, while $\varepsilon_{1}$ is the factor for inexistence of the solid-solid connection and $\varepsilon_{2}$ is for the existence of solidsolid connection and solid-fluid connection.

Actually, in the microspace structure of porous medium materials, the existence of ideal even distribution of the pores in the porous body is impossible. So there is a big error between the ideal model mentioned above and the real body. The available ideal models and empirical equations for foam thermal insulating materials are only generally related to pores proportion, which is the approximate reflection of the apparent thermal conductivity in the macrospace. But for the real foam material whose pore distribution is irregular, the available ideal models and empirical equations are not relative to the microstructure and cannot expose the actual heat and mass transfer procedure and the distribution of temperature and humidity. As a result, the big error is presence in the research work.

The other method involves fractal theory. Fractal theory, introduced into the estimation and research work to calculate the thermal conductivity of porous foam materials, is a new way for the theory development on thermal performance of porous medium materials. Fractal theory was first put forward in 1975 by Mandelbrot who was a professor from Harvard University in USA. Some experts, such as Pitchumani [9], Yu and Li [5], and Ma et al. [6], have done deep researches on the effective thermal conductivity of granular porous medium by fractal theory and have created corresponding mathematical equations. Based on the fractal theory, Thovert et al. [10], Zhang et al. [11], and so on developed the theoretical models for calculation of the effective thermal conductivity of irregular porous medium. According to the concept of Sierpinski carpet model, Pitchumani and Ramakrishnan [12, 13] created the pore distribution theoretical model, but the model and mathematical equations were very complicated in fractal dimension. Ma et al. [6] built a mathematical model of effective thermal conductivity for porous medium according to fractal theory, which manifested that the thermal conductivity of porous medium was a function of the pore ratio, the area ratio, the thermal conductivity ratio in components, and the thermal contact resistance all together. It had nothing to do with empirical constants and less parameters and simple to calculate in the formula. However, different porous media are not the same with each other in internal fractal essence. And it is also difficult to estimate the thermal contact resistance of porous medium in practice. The universality of the model still needs to be further verified. Thovert et al. [10] illuminated the fractal porous medium by the percolation mathematical model, and did the solution by geometrical iteration. Whereafter, Adler, Thovert, and Thompson added empirical constants gotten by experiments into Adler's function. And the function is commonly described as

$$
\lambda=\lambda_{0} \phi^{\alpha}
$$

where $\lambda_{0}$ is the thermal conductivity of the liquid in the porous material pores $\left(\mathrm{Wm}^{-1} \mathrm{~K}^{-1}\right)$. And the superscript $\alpha$ here is defined as:

$$
\alpha=\frac{d_{s}+D_{f}\left(2-d_{s}\right)}{d_{s}\left(3-D_{f}\right)}
$$

where, the fractal dimension factor $D_{f}=2.5-2.85$, and spectral dimension $d_{s}$ is used to describe the procedure of the percolation in the pores.

Yangsheng [14], based on the percolation theory, created the relationship between the pore diameters of various grain material and the thermal conductivity. But the pore porosity, the fractal dimension, and the microstructure are not involved in the model. Pitchumani and Yao [15] calculated the transverse and longitudinal fractal dimensions to illuminate the microstructure fibrous materials, and the thermal conductivity was derived based on the conventional heat transfer theory. But the model only serves some certain fibrous porous materials well.

So, it is considerably difficult and also unpractical to build a theoretically mathematical model of effective thermal conductivity that is universal for porous medium. Consequently, crating a mathematical model of thermal conductivity for one certain porous medium that reflects its structure characteristic in internality, is an important developing direction for the porous medium research work.

\section{Microstructures of the Open Cell Polyurethane Foam and Fractal Description}

2.1. Microstructures. The open cell polyurethane is composed of solid substrates and cells. By the effect of foaming agent and cell opening agent, great deals of cells are generated and continuously distributed inside the material. The cells connect with each other side by side, and the gas in pores can flow freely through one cell to another. That is really an advantage to expulse foaming agent and vapors that embraced in pores. Meanwhile, the gas in pores can easily be expelled as the thorough connection of cells. The solid substrate of open cell polyurethane has the certain intensity to support the material and to prevent the collapse in vacuum state. So the polyurethane foam with open cell structure can be used widely as the core material of the vacuum insulation panel.

Microstructures of the open cell polyurethane, consisting of skeleton of solid substrate (the white part in the picture) and cells (the black part in the picture), is shown in Figure 1 (taken by electron microscopy). Cells are generally cubed structurally in the space and continuously distributed in section plane, and the dimensions of the apertures are in 

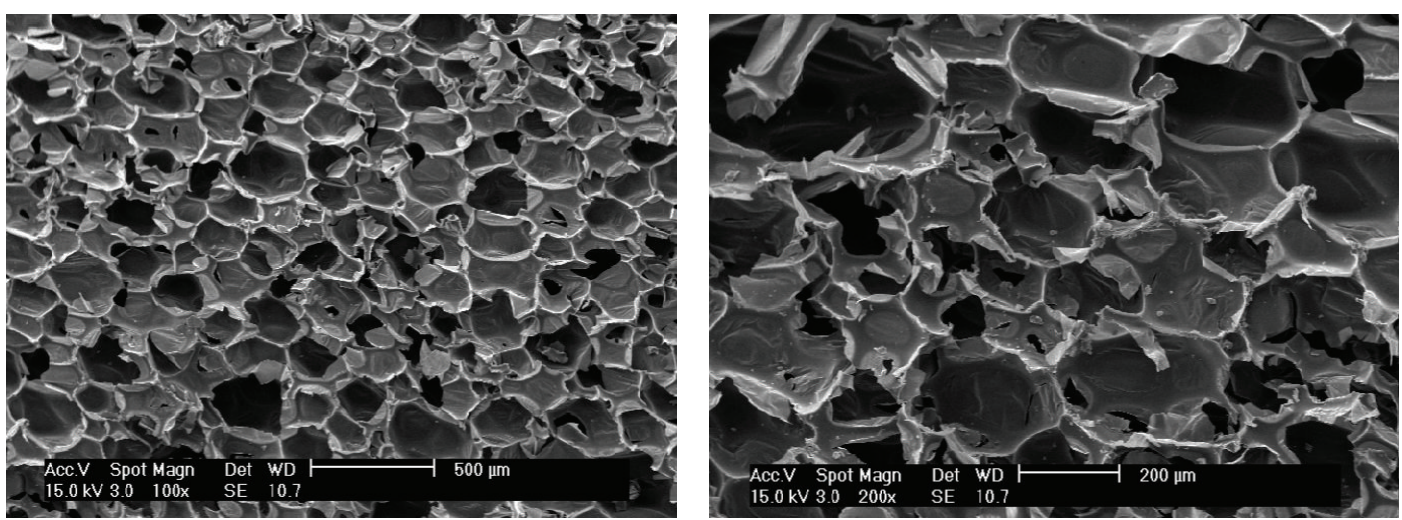

Figure 1: Microstructure section of the open cell polyurethane foam (magnified 500 and 200 times).

range of $140-220 \mu \mathrm{m}$ and the length of average skeleton is $125 \mu \mathrm{m}$. The cells' dimensions are various and the distribution is random and irregular.

2.2. Fractal Description. Fractal theory, since it was born, has attracted lots of scientists' interest because of its unique advantages of research in irregular and complicated objects geometrically and success in dealing with many problems of geometry, physics, geology, hylology, and so on. Meanwhile, the various problems in the scientific subjects also enhanced the development of the fractal theory. Fractal theory is an effective approach to describe nonlinear phenomena in nature, complicated structures in geometry, and internal objects and spatial distribution. Fractal theory firstly committed research on nonlinear complex systems and analyzed the inner laws from the investigated subjects that were not simplified and abstract. That is essential distinction between fractal theory and linear way. Two subjects can be treated as the self-similarity, while the fractal dimension values are equal, according to the fractal theory. Various fractal models have been built for porous media materials by experts and researchers, and the famous models, such as Sierpinski carpet model, Menger sponge model, and Koch curve model, are adopted by many researchers. However, almost porous media materials in nature are not the same with the models mentioned above. They are not strict similarity but similar in mathematical calculation.

According to fractal theory, it is a self-similar scaling relationship between metric measure of objects $\delta$ and physical quantity $N(\delta)$ existed in $D_{f}$ dimension Euclidean space including area and volume, or length of a porous fractal [16]:

$$
N(\delta) \propto \delta^{D_{f}} .
$$

For one fractal body, the fractal dimension value $D_{f}$ is in the range from 2 to 3 . But for the microstructure open cell polyurethane, the diameters of the pores are different. The structure is irregular, and the distribution is random. For the open cell polyurethane foam, the largest pore size of cells is $D_{\text {max }}=220 \mu \mathrm{m}$, and the smallest one is $D_{\text {min }}=140 \mu \mathrm{m}$; and supposing the measure length $\delta$ for the pace $D_{\max } \rightarrow D_{\min }$, the cell volume $V(\delta)$ can be described in the following:

$$
V(\delta) \propto \delta^{D_{f}} .
$$

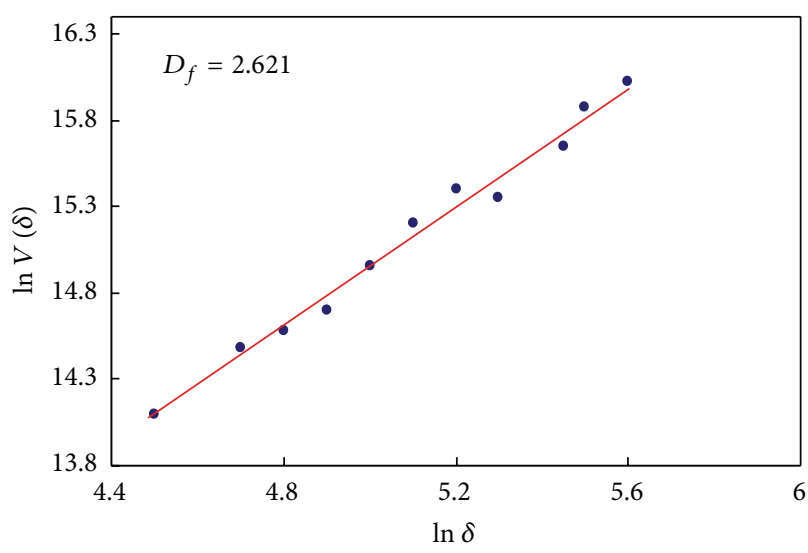

FIGURE 2: Fractal dimension calculation for cell body of the open cell polyurethane foam.

Based on fractal theory, the cell distribution has selfsimilarity statistically for the open cell polyurethane foam. Equation (6) can be replaced by the following:

$$
V(\delta)=C \delta^{D_{f}},
$$

where $C$ is constant. And taking the logarithm to (7), (8) can be gotten as

$$
\ln V(\delta)=\ln C+D_{f} \ln \delta .
$$

According to Sierpinski carpet's random fractal method, Figure 1 is fractal calculated and the result was shown in Figure 2. That is, the open cell polyurethane foam volume in this research has the fractal feature and the fractal dimension value is $D_{f}=2.621$ for the sample.

However, the structure in the porous medium is irregular and the distribution of the pore is also random. The physical quantity $N(\delta)$, the pores' quantity, has the relationship with $\delta$ and the pore diameter $D$. So (5) can be rewritten as

$$
N(\delta>D)=\left(\frac{D_{\max }}{D}\right)^{D_{f}}
$$

or

$$
N^{\prime}\left(\delta>D_{\min }\right)=\left(\frac{D_{\max }}{D_{\min }}\right)^{D_{f}} .
$$



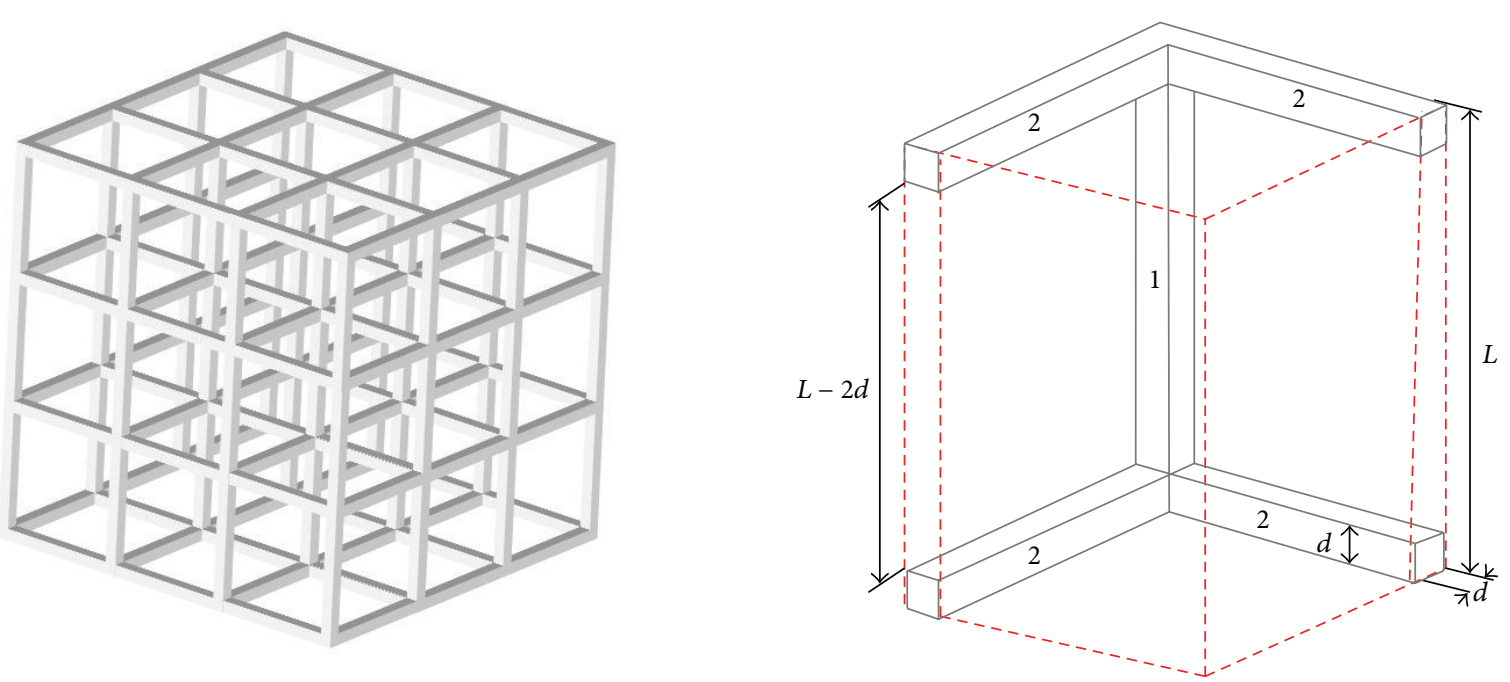

FIGURE 3: Simplified structure model of the open cell polyurethane foam.

Taking differential coefficient to (9), then

$$
d N=-D_{f} D_{\max }^{D_{f}} D^{-D_{f}-1} d D
$$

So, combining with (10) and (12) can be gotten as

$$
-\frac{d N}{N^{\prime}}=D_{f} D_{\min }^{D_{f}} D^{-\left(D_{f}+1\right)} d D
$$

Here, the pore distribution probability function $f(D)=$ $D_{f} D_{\min }^{D_{f}} D^{-\left(D_{f}+1\right)}$ can be rewritten as

$$
\int_{-\infty}^{\infty} f(D) d D=\int_{D_{\min }}^{D_{\max }} f(D) d D=1-\left(\frac{D_{\min }}{D_{\max }}\right)^{D_{f}} .
$$

The fractal effective diameter $L$ of the pores in the open cell polyurethane can be calculated according to the pore distribution probability function:

$$
\begin{aligned}
L & =\int_{D_{\min }}^{D_{\max }} D D_{f} D_{\min }^{D_{f}} D^{-\left(D_{f}+1\right)} d D \\
& =\frac{D_{f}}{D_{f}-1} D_{\min }\left[1-\left(\frac{D_{\min }}{D_{\max }}\right)^{D_{f}-1}\right] .
\end{aligned}
$$

Based on the inner structure of the open cell polyurethane form, we suppose that cells are cubed and well distributed, as in Figure 3.

\section{The Equivalent Thermal Conductivity of Fractal Model}

The equivalent thermal conductivity $\lambda_{e}$ of the porous open cell medium materials is the function of the variable thermal conductivities of the phases, the inner structure, and the pores distribution [17]. So the equivalent thermal conductivity $\lambda_{e}$ can be illuminated in the following:

$$
\lambda_{e}=f\left(\sum \lambda_{i}, \varphi, D_{f}\right)
$$

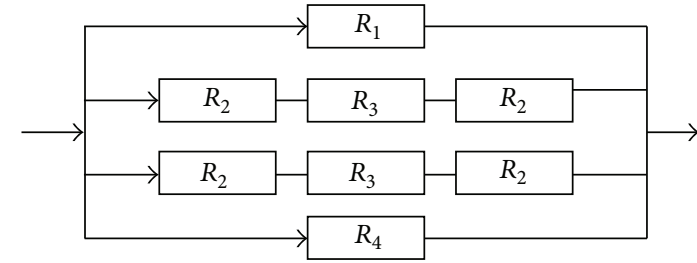

FIGURE 4: Thermal network sketch.

where $\lambda_{i}$ is thermal conductivity of the phase $i$ in the porous medium materials. For the solid phase, the conductivity is $\lambda_{s}$, while $\lambda_{g}$ for the gas in the pores $\varphi$ is the porosity of the average volume.

The mathematic model for the open cell polyurethane is developed on the basis of (15) in this paper. Neglecting the effect of heat radiation in cells and gas heat convection, we conclude that the heat transfer in one cell of open cell polyurethane form is only influent by adjacent cells. For one cell, we suppose that the structure is regular prism, the fractal diameter $L$; the height is mentioned above in (14), while the solid substrates height $d$, just as in Figure 3. So, the whole heat transfer procedure in the cell can be analyzed as the electricity transfer in the electrocircuit. Suppose that heat current flow from top to underside through the cell body, then thermal resistance of the cell mainly consists of four parts.

$R_{1}$ is thermal resistance of vertical pillar $1, R_{2}$ level pillar $2, R_{3}$ gas among level pillars, and $R_{4}$ gas in cavity.

The thermal resistance simplified model can be described as in Figure 4.

According to interrelated heat transfer knowledge, we can easily get that

$$
\begin{gathered}
R_{1}=\frac{L}{\lambda_{s} d^{2}} \\
R_{2}=\frac{2 d}{\lambda_{s} d(L-2 d)}=\frac{2}{\lambda_{s}(L-2 d)}
\end{gathered}
$$




$$
\begin{gathered}
R_{3}=\frac{(L-2 d)}{\lambda_{g}(L-2 d) d / 2}=\frac{2}{\lambda_{g} d} \\
R_{4}=\frac{4 L}{\lambda_{g}(L-2 d)^{2}} \\
R_{\text {total }}=\frac{4 L}{\lambda_{e} L^{2}}=\frac{4}{\lambda_{e} L},
\end{gathered}
$$

where $R_{\text {total }}$ is entire thermal resistance; $\lambda_{s}$ is thermal conductivity of foam's skeleton; $\lambda_{g}$ is thermal conductivity of gas in cells; $\lambda_{e, \text { foam }}$ is effective thermal conductivity of the form.

From the analysis above, we can deduce that

$$
R_{\text {total }}=\frac{R_{1} R_{4}\left(2 R_{2}+R_{3}\right)}{2 R_{1} R_{4}+\left(2 R_{2}+R_{3}\right)\left(R_{1}+R_{4}\right)} .
$$

From (3) and (17), (18) can be easily gotten:

$$
\lambda_{e, \text { foam }}=\frac{4 \lambda_{s} d^{2}}{L^{2}}+\frac{\lambda_{g}(L-2 d)^{2}}{L^{2}}+\frac{4 \lambda_{g} \lambda_{s}(L-2 d) d}{L\left(2 \lambda_{g} d+L \lambda_{s}-2 \lambda_{s} d\right)},
$$

where $\lambda_{e \text {,foam }}$ in (18) is the effective thermal conductivity when there is static gas in pores of the open cell polyurethane.

The conception of porosity $\varphi$ for porous polyurethane would be introduced here. Generally, it is the ratio of the summation of the vacancy volume $V_{i}$ to the whole material block volume $V$. With the calculating methods by the fractal theory, the porosity $\varphi$ can be easily illuminated as [18]

$$
\varphi=\frac{\sum_{i=1}^{n} V_{i}}{V}=\left(1-\frac{2 d}{L}\right)^{D_{f}} .
$$

Combining (18) with (19), the effective thermal conductivity will be gotten:

$$
\begin{aligned}
\lambda_{e, \text { foam }}= & \lambda_{s}\left(1-\varphi^{1 / D_{f}}\right)^{2}+\lambda_{g} \varphi^{2 / D_{f}} \\
& +2 \frac{\lambda_{g} \lambda_{s}\left(1-\varphi^{1 / D_{f}}\right) \varphi^{1 / D_{f}}}{\lambda_{g}\left(1-\varphi^{1 / D_{f}}\right)+\lambda_{s} \varphi^{1 / D_{f}}} .
\end{aligned}
$$

From (20), we can conclude that the effective thermal conductivity of the open cell polyurethane form has relationship with the phases of the cell body and the fractal dimension and the cell structure, that is, the porosity.

The thermal conductivity would decrease with fractal dimension's increase of cells volume and increase of pore porosity, and that is in accordance with heat conducting performance. The bigger the fractal dimension and porosity are, the less the solid substrates are and the worse the heat conducting property is.

\section{The Effective Thermal Conductivity of Thermal Radiation}

Heat radiation is an important factor for the open cell polyurethane foam. It can be treated as a gray-body medium to estimate the radiation heat flow in cells [10]. So the rate of radiation heat flow for a cell is

$$
q_{r}=-\frac{4 \sigma\left(T_{1}^{4}-T_{2}^{4}\right)}{3 \beta L},
$$

where $\sigma$ is Stefn-Boltzmann constant, $\sigma=5.6697 \times$ $10^{-8} \mathrm{~W} /\left(\mathrm{K}^{4} \cdot \mathrm{m}^{2}\right), \beta$ is radiation extinction coefficient for porous medium, and $T_{1}$ and $T_{2}$ are separately heat flow's temperature of entrance and exit.

So we can get the equivalent radiation thermal conductivity $\lambda_{e, r}$ for a porous medium:

$$
\lambda_{e, r}=\frac{4 \sigma\left(T_{1}^{2}+T_{2}^{2}\right)\left(T_{1}+T_{2}\right)}{3 \beta} .
$$

\section{The Comparison between Results of Theoretical Calculation and Experiment}

The entire equivalent thermal conductivity $\lambda_{e}$ can be obtained in (23) on the condition of integrating the heat conducting and radiation-conduction heat transfer together:

$$
\lambda_{e}=\lambda_{e, \text { foam }}+\lambda_{e, r}
$$

The certain open cell polyurethane foam above is selected as a sample to test in experiments, and its thermal conductivity of solid substrates is $\lambda_{s}=0.5832 \mathrm{~W} /(\mathrm{m} \cdot \mathrm{K})$, the thermal conductivity of gas in pore is $\lambda_{g}=0.0229 \mathrm{~W} /(\mathrm{m} \cdot \mathrm{K})$, and the decay coefficient tested is $\beta=445 \mathrm{~m}^{-1}$. The measurement way to test thermal conductivity of the sample is heat guarded plate method. And the standard of test refers to GB/T33992009. The results are collected in Table 1.

\section{Conclusion}

It can be found from Table 1 that there is little difference between the results calculated by the theoretical model present above and the experimental ones. Conclusions from the research work are as follows.

There is a good consistency between experimental and theoretical calculations presented in this paper. Error is less than $5 \%$. Especially when taking the open cell polyurethane foam as the core of vacuum insulation panels, the gas thermal conductivity in (18) can be ignored, and calculations simplified and more accurate results can be obtained.

The effective thermal conductivity of the open cell polyurethane foam has a relationship with the material properties, inner microstructure, and the service environmental temperature. Thermal conductivity during heat conduction in entire effective thermal conductivity is predominant in normal temperature, while the effective thermal conductivity during radiation is a little undulating, but the value is not primary. So, increasing the porosity of the body can enhance its entire heat insulating property, on conditioning that its structural strength is enough for the open cell polyurethane foam.

The research work has manifestly established a connection between a thermophysical property and the internal 
TABLE 1: The comparative table between results of calculation and experiment.

\begin{tabular}{|c|c|c|c|c|c|c|c|c|c|}
\hline \multirow{2}{*}{ Sample } & \multirow{2}{*}{$\begin{array}{l}\text { Density } \\
\mathrm{kg} / \mathrm{m}^{3}\end{array}$} & \multirow{2}{*}{$\begin{array}{c}\text { Porosity } \\
\% \\
\end{array}$} & \multirow{2}{*}{$\begin{array}{c}\text { Fractal } \\
\text { dimension }\end{array}$} & \multirow{2}{*}{$\begin{array}{c}\text { Average } \\
\text { temperature K }\end{array}$} & \multicolumn{4}{|c|}{$\lambda \mathrm{W} /(\mathrm{m} \cdot \mathrm{K})$} & \multirow{2}{*}{$\begin{array}{c}\text { Difference } \\
\%\end{array}$} \\
\hline & & & & & $\lambda_{e, \text { foam }}$ & $\lambda_{e, r}$ & $\lambda_{e}$ & $\lambda_{\text {test }}$ & \\
\hline \multirow{2}{*}{1} & \multirow{2}{*}{45} & \multirow{2}{*}{81} & \multirow{2}{*}{2.63} & 300 & 0.2804 & 0.0022 & 0.2826 & 0.280 & $-0.93 \%$ \\
\hline & & & & 355 & 0.2804 & 0.0028 & 0.2832 & 0.287 & $1.3 \%$ \\
\hline \multirow{2}{*}{2} & \multirow{2}{*}{60} & \multirow{2}{*}{72} & \multirow{2}{*}{2.53} & 300 & 0.3186 & 0.0022 & 0.3208 & 0.330 & $2.8 \%$ \\
\hline & & & & 355 & 0.3186 & 0.0028 & 0.3214 & 0.332 & $3.2 \%$ \\
\hline
\end{tabular}

microstructure of porous media by fractal theory. The theoretical work would be an important reference in enhancing heat insulating of porous media and useful in developing new material for environmental protection and energy conservation.

\section{Nomenclature}

C: $\quad$ Constant value

$D_{\min }$ : The smallest bore size in dimension

$D_{\text {max }}$ : The biggest bore size in dimension

$D_{f}$ : Fractal dimension factor

$d_{s}: \quad$ Spectral dimension

$d$ : Width of the model pillar

$L: \quad$ Length of the model pillar

$N(\delta)$ : Physical quantity

$R: \quad$ Thermal resistance $\left(\mathrm{m}^{2} \cdot \mathrm{K} / \mathrm{W}\right)$

$T: \quad$ Temperature $(\mathrm{K})$

$V: \quad$ Volume $\left(\mathrm{m}^{3}\right)$.

\section{Greek Symbols}

$\alpha: \alpha=\left(d_{s}+D_{f}\left(2-d_{s}\right)\right) / d_{s}\left(3-D_{f}\right)$

$\sigma$ : Stefn-Boltzmann constant,

$$
\sigma=5.6697 \times 10^{-8} \mathrm{~W} /\left(\mathrm{K}^{4} \cdot \mathrm{m}^{2}\right)
$$

$\beta$ : Radiation extinction coefficient

$\lambda$ : Thermal conductivity $(\mathrm{W} /(\mathrm{m} \cdot \mathrm{K}))$

$\delta$ : Variable measure length $(\mathrm{m})$

$\varphi$ : Pore porosity in the average volume.

\section{Subscripts and Superscripts}

$\begin{array}{ll}e: & \text { Effective } \\ r: & \text { Radiation } \\ g: & \text { Residual gaseous phase in the pore } \\ f: & \text { The fluid phase } \\ S: & \text { The solid phase } \\ \text { total: } & \text { Total value }\end{array}$

test: The value gotten from experiments.

\section{Acknowledgment}

This work was financially supported by Science \& Technology Program of Shanghai Maritime University no. 20120091. We are grateful to Professor Wenzhe Sun and Professor Dan Cao for their advices and suggestions for this project. The authors also acknowledge Dr. Wenzhong Gao with valuable discussion and contributions in mounting the experimental and installing the data acquisition devices.

\section{References}

[1] Y. Chen and M. Shi, "Determination of effective thermal conductivity for porous media using fractal techniques," Journal of Engineering Thermophysics, vol. 20, no. 5, pp. 608-615, 1999.

[2] S. Whitaker, "Diffusion and dispersion in porous media," AIChE Journal, vol. 13, no. 6, pp. 1066-1085, 1967.

[3] S. Whitaker, "Advances in the theory of fluid motion in porous media," Chemical Engineering, vol. 61, pp. 14-28, 1969.

[4] S. Whitaker and W. T.-H. Chou, "Drying granular porous media-theory and experiment," Drying Technology, vol. 1, no. 1, pp. 3-33, 1983.

[5] B. Yu and J. Li, "Some fractal characters of porous media," Fractals, vol. 9, no. 3, pp. 365-372, 2001.

[6] Y. Ma, B. Yu, D. Zhang, and M. Zou, "A self-similarity model for effective thermal conductivity of porous media," Journal of Physics D, vol. 36, no. 17, pp. 2157-2164, 2003.

[7] A. Lagarde, "Consideration sur le transfert de chaleur en milieu poreux," Institut Francais Du Petrole, vol. 2, pp. 383-446, 1965.

[8] J. K. Williams and R. A. Dawe, "Fractals-an overview of potential applications to transport in porous media," Transport in Porous Media, vol. 1, no. 2, pp. 201-209, 1986.

[9] R. Pitchumani, "Evaluation of thermal conductivities of disordered composite media using a fractal model," Journal of Heat Transfer, vol. 121, no. 1, pp. 163-166, 1999.

[10] J. F. Thovert, F. Wary, and P. M. Adler, "Thermal conductivity of random media and regular fractals," Journal of Applied Physics, vol. 68 , no. 8, pp. 3872-3883, 1990.

[11] D. Zhang, H. Yang, and M. Shi, "Important problems of fractal model in porous media," Journal of Southeast University, vol. 32, no. 5, pp. 692-697, 2002.

[12] R. Pitchumani and B. Ramakrishnan, "A fractal geometry model for evaluating permeabilities of porous preforms used in liquid composite molding," International Journal of Heat and Mass Transfer, vol. 42, no. 12, pp. 2219-2232, 1999.

[13] B. Ramakrishnan and R. Pitchumani, "Fractal permeation characteristics of preforms used in liquid composite molding," Polymer Composites, vol. 21, no. 2, pp. 281-296, 2000.

[14] Z. Yangsheng, Coupling Effect and Engineering Reflection of Porous Media Materials, Science Press, Beijing, China, 2010.

[15] R. Pitchumani and S. C. Yao, "Correlation of thermal conductivities of unidirectional fibrous composites using local fractal techniques," Journal of Heat Transfer, vol. 121, no. 1, pp. 788-796, 1991.

[16] B. B. Mandelbrot, The Fractal Geometry of Nature, W.H. Freeman, New York, NY, USA, 1983. 
[17] A. K. Kan, T. T. Zhang, and H. J. Lou, "Fractal study of effective thermal conductivity of fiber glass materials," Chinese Journal of Vacuum Science and Technology, vol. 33, pp. 654-660, 2013.

[18] Z. Donghui, J. Feng, and S. Mingheng, "Heat conduction in fractal porous media," Journal of Applied Sciences, vol. 21, no. 3, pp. 253-2257, 2003. 

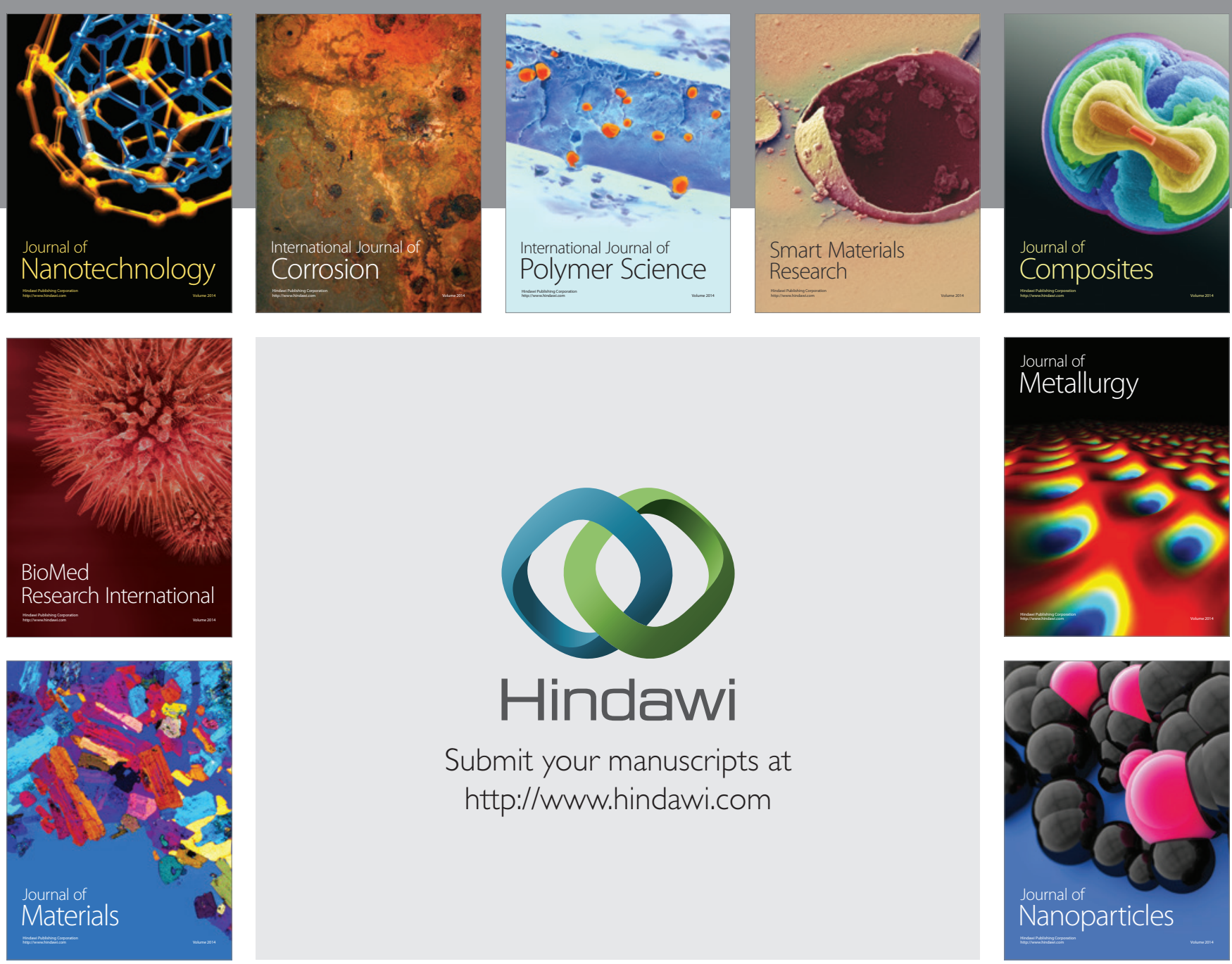

Submit your manuscripts at http://www.hindawi.com
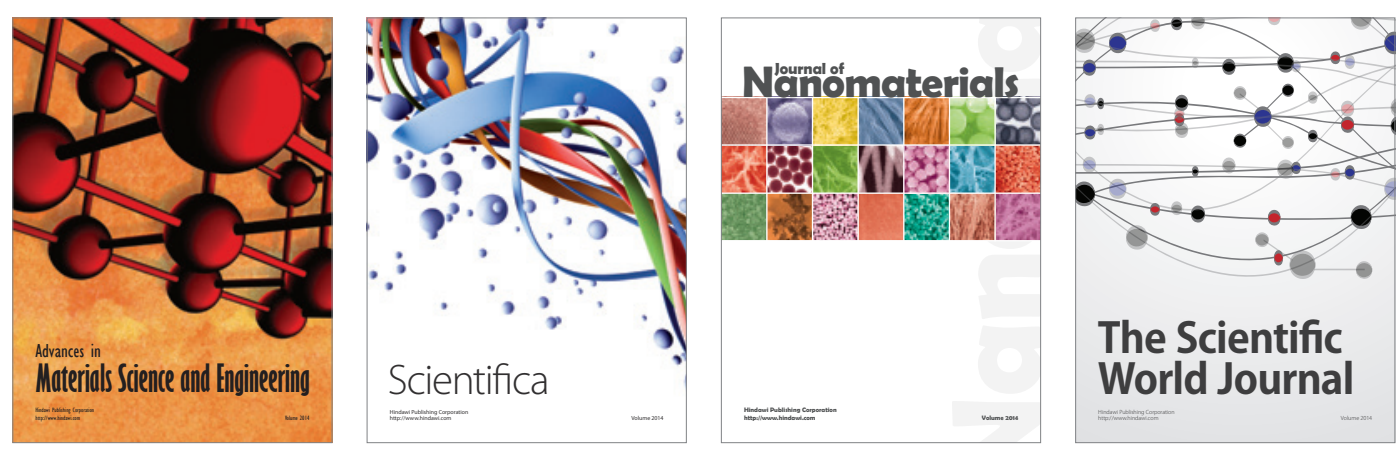

\section{The Scientific World Journal}
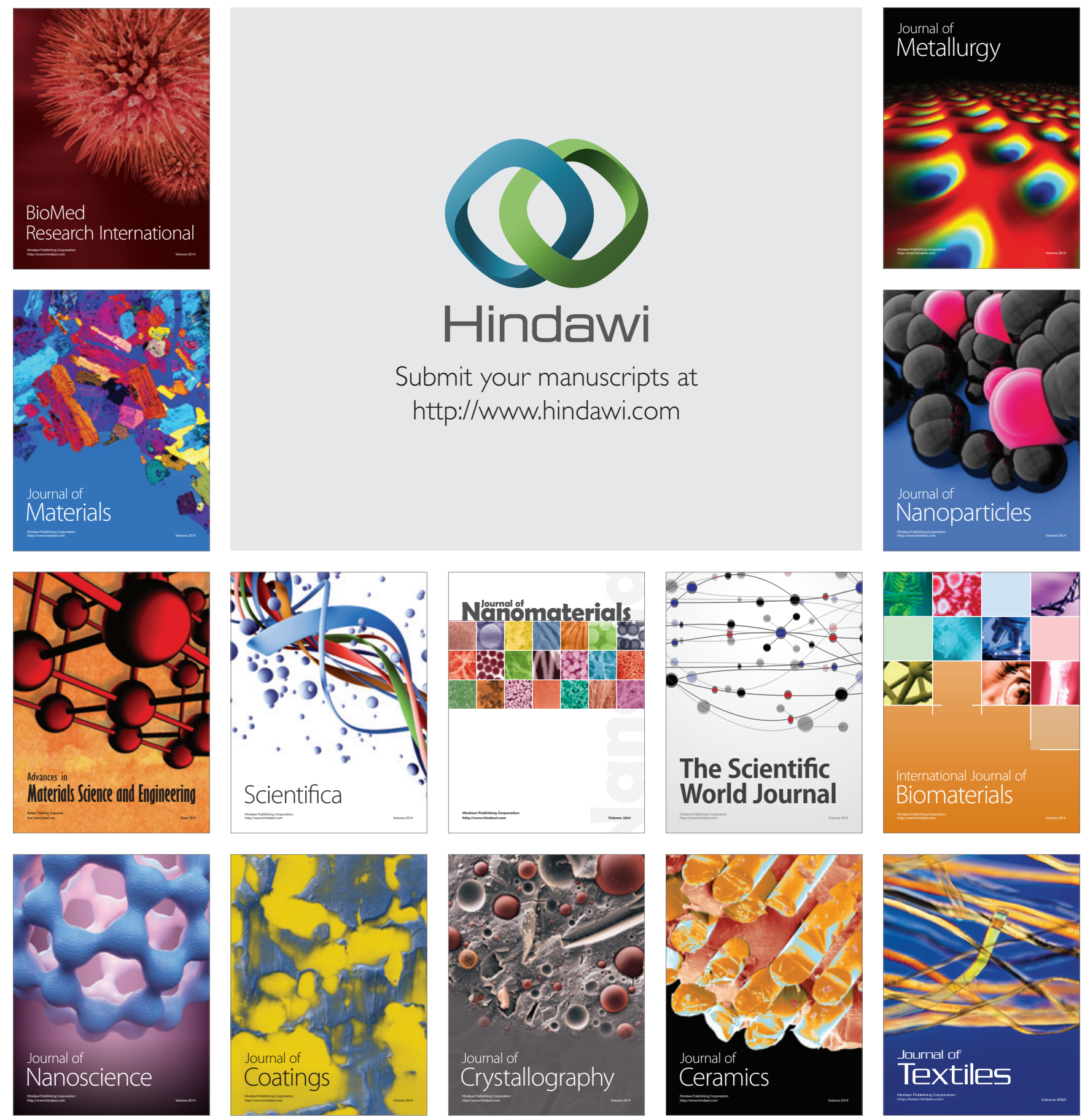